\title{
Bottom Reflectance Influence on a Color Correction Algorithm for Underwater Images
}

\author{
Julia Åhlén ${ }^{1}$ and David Sundgren ${ }^{2}$ \\ 1 Centre for Image Analysis, \\ Uppsala University \\ 2 Department of Numerical Analysis and Computer Science, \\ Stockholm University
}

\begin{abstract}
Diminishing the negative effects of water column introduced on digital underwater images is the aim of a color correction algorithm presented by the authors in a previous paper. The present paper describes an experimental result and set of calculations for determining the impact of bottom reflectance on the algorithm's performance. This concept is based on estimations of the relative reflectance of various bottom types such as sand, bleached corals and algae. We describe the adverse effects of extremely low and high bottom reflectances on the algorithm.
\end{abstract}

\section{Introduction}

The examination of underwater images is important for marine scientists. In some cases proper color representation is important, such as studies of archaelogical sites in the oceans. Another application is the observation of corals bleaching. Light that enters the ocean will be reflected, transmitted and scattered by floating biological organisms. High energy wavelengths will not penetrate deeper than approximatly 10 meters according to severe absorbtion by water molecules. Thus images produced in underwater environments tend to be bluish. When constructing a color correction algorithm for underwater environments one has to take into consideration the apparent optical properties of the water column such as absorbtion and scattering effects. An optimization technique [3] can be applied to spectral images to retrive underwater information without a priori knowledge of the optical properties of the water column or bottom reflectivity or depth. However such methods may be appropriate for studies of hyperspectral images with a broad range of spectral channels measured with reliable instruments.

In this study, using a digital still camera for data collection, we significantly decrease the economical cost for such operations. The drawback is that we are limited to 3 spectral channels. The restauration of bluish images is based on the exact depth measurements and retreived diffuse attenuation coefficients for those depths [1]. The reflectance calculations require a few assumptions to be made. In $60 \%$ of the tested images the algoritm is found to work well.

It was noticed that extremely high or low values of the bottom reflectance have a negative effect on the algorithm. However by introducing a bottom reflectance compensation function to the model this negative effect is reduced considerably. We are focusing on shallow waters with various bottom types. 


\section{A Model for Color Correction of Underwater Images}

The reflectance of an object can be considered as a ratio of the intensity of reflected radiant energy to that reflected from a defined reference standard. In image collection and further processing we are using a grey Spectralon [2] as such a standard. The grey plate is placed on a surface next to the coral or other target. We assume that the Spectralon is taking in as much light as the surrounding environment. In [1] we calculate the reflectance of various bottom types in each channel as in equation (1).

$$
R_{r}=\frac{I}{L_{\text {in }}}=\frac{I R}{L_{\text {out }}},
$$

where $R_{r}$ is the reflectance of the subject in the red channel, $L_{\text {in }}$ is the incident radiation (which is the same as on the the Spectralon), $I$ is the intensity of a pixel and $L_{\text {out }}$ is the intensity value of a Spectralon in the image.

The $K_{d}$ (attenuation coefficient) gives us a rate at which each wavelength is absorbed by the water column. Since we are limited to 3 spectral channels we do not need to calculate it for each $\lambda$, thus for each depth we are choosing an appropriate wavelength to work with. However the experimental data showed that $K_{d}$ values do not increase strictly with depth. This is due to lack of light which affected the red channel most and makes it the noisiest one. In the model we can estimate the new values for the image taken at $z$ meters depth as if it was taken at $z_{1}$ meters depth with equation (2), which is derived from Beer's law.

$$
I\left(z_{1}\right)=I(z) e^{K_{d}(z) z-K_{d}\left(z_{1}\right) z_{1}} .
$$

Where $I(z)$ is the pixel intensity in the image for depth $z$.

\section{Compensation of Bottom Reflectance on the Images}

The presence of bottom reflected light makes color correction for underwater environments easier. Extreme bottom reflectances, however, has the effect of making the reflectance of objects in the image appear different from their actual values. The low performace of the algorithm on images with extremely low or high bottom reflectances is largely due to this effect and we suggest a method to compensate for it.

Since the Spectralon that we are using as a reference target in each image has a known reflectance profile and the incoming light decreases with depth according to Beer's law the intensity of a pixel should be proportional to the incoming light. In our study we have discovered that camera behaviour affects the real outcome less than the bottom reflectance. We compare intensity profiles of pairs of images at the same depth but with different bottom types. The grey reference target has high deviations in intensity profile from pair to pair. This indicates that the images with intensities abnormally higher than a corresponding pair can be considered noisy due to bottom reflectance variations. 


\subsection{Compensation function}

As a first step in developing a compensation function we calculate the incoming light at the surface and estimate the remaining light at the actual depth. This is possible since we already have $K_{d}$ values for each depth. The apparent reflectance of the Spectralon as a function of bottom reflectance can be expressed as $R_{\text {as }}=$ $R+f\left(R_{\text {bottom }}\right)$. This is valid for any object in an image since we assume that light is distributed equally on the whole image. Thus we can estimate the effect of bottom reflectance on any object given that the underwater environment is of the same type as in the reference image.

The final step in producing the compensation function is to use a sequence of images with varying bottom reflectance from the same depth to obtain the apparent spectralon reflectance based on an estimation of incoming light. Bottom reflectances were plotted against the apparent reflectance as in figures 1,2 and 3.

The compensating function $f\left(R_{\text {bottom }}\right)=R_{\text {as }}-R$ is then easily obtained from these values.

We introduce the compensation function by replacing $I_{z}$ in equation 2 with $\frac{\left(R_{r}-f\left(R_{\text {bottom }}\right)\right) L_{\text {out }}}{R}$ where $R_{r}$ is the reflectance of a typical object in the image, $f\left(R_{\text {bottom }}\right)$ is the function that describes how the bottom reflectance affects the apparent reflectance of the object, $L_{\text {out }}$ is the pixel intensity value and $R$ is the measured reflectance of the Spectralon.

$$
I\left(z_{1}\right)=\frac{\left(R_{r}-f\left(R_{\mathrm{bottom}}\right)\right) L_{\mathrm{out}}}{R} e^{K_{d}(z) z-K_{d}\left(z_{1}\right) z_{1}} .
$$
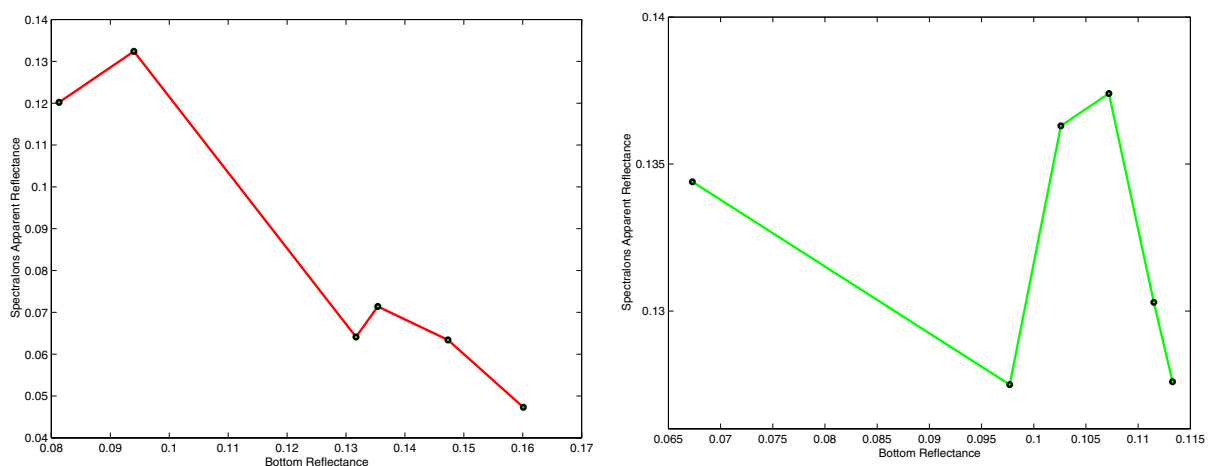

Fig. 1. Apparent reflectance of Spectralon as a function of bottom reflectance in the red channel.

Fig. 2. Apparent reflectance of Spectralon as a function of bottom reflectance in the green channel. 


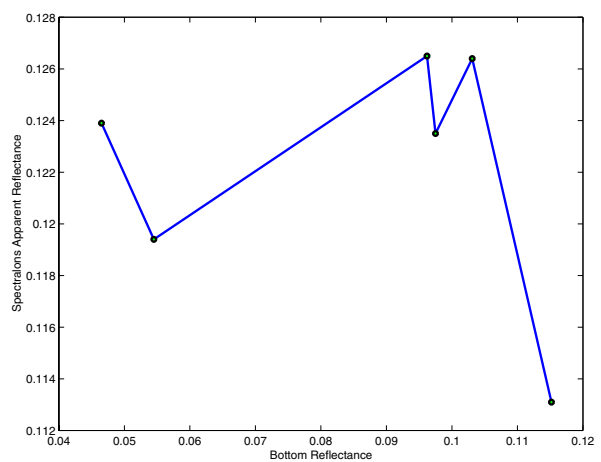

Fig. 3. Apparent reflectance of Spectralon as a function of bottom reflectance in the blue channel.

\section{Results and Discussion}

Using an improved model for color correction of underwater images we are able to remove the negative effects of water column properties always present on underwater images almost irrespective of bottom reflectance. We point out that bottom reflectance is not the only factor that could contribute to poor results of the algorithm. Such factors could be unpredictible behaviour of the CCD sensor, difficulties obtaining the exact depth and measurements of incoming light.

As we are using only a digital camera and a Spectralon our calculations are not exact. However this method is quite inexpensive yet reasonably accurate. After introducing the compensating function into the equation we could restore the colors in $80 \%$ of the images.

We show the result on an image with high bottom reflectance featuring brown algae, figure 4 . Figure 5 is the image corrected without compensation for bottom reflectance and figure 6 is corrected and compensated for bottom reflectance with the compensation function described above.

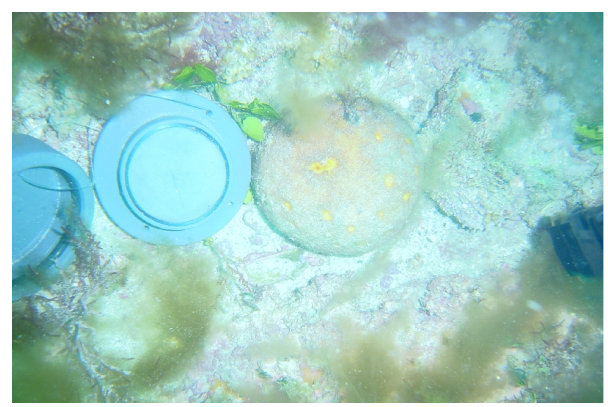

Fig. 4. Original image with high bottom reflectance.

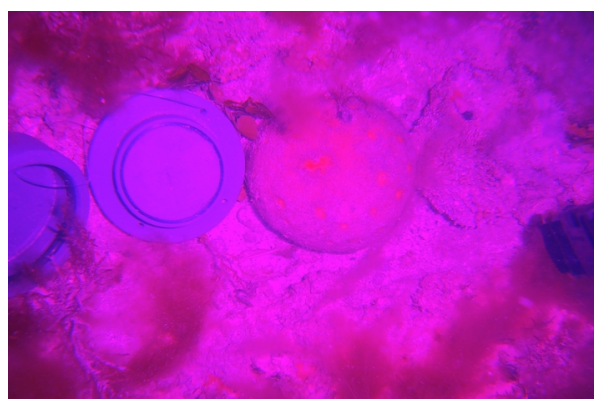

Fig. 5. Image corrected without reflectance compensation. 


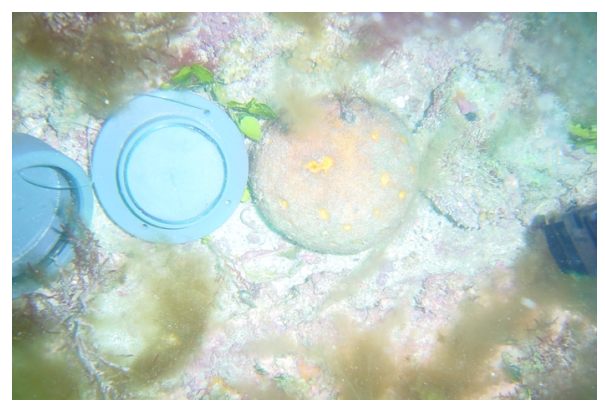

Fig. 6. Image corrected with reflectance compensation function.

\section{Acknowledgements}

We would like to thank Robert Steward and David English for their help in collecting the images, and Ewert Bengtsson and Tommy Lindell for many useful discussions. This work was supported by the KK-foundation.

\section{References}

1. J. Åhlén, E. Bengtsson, and T. Lindell. Color correction of underwater images based on estimation of diffuse attenuation coefficients. January 2003.

2. Labsphere. Spectralon: Reflectance material for component fabrication, November 2002.

url: http://www.labsphere.com/products/Products.asp

3. Z. Lee, K.L. Carder, R.F. Chen, and T.G. Peacock. Properties of the water column and bottom derived from airborne visible infrared imaging spectrometer (aviris) data. Journal of Geophysical Research, Vol. 106, No. C6, pages 11639-11651, 2001. 